\title{
Actitud sobre la maternidad adolescente a partir de artículos de prensa digital venezolana y francesa
}

Appraisal-related elements of attitude in the discourse of Venezuelan and French digital news about teenage pregnancy

Yarubi Díaz Universidad de Los Andes, Facultad de Humanidades y Educación

Melva Márquez Universidad de Los Andes, Facultad de Humanidades y Educación

\section{Resumen}

El objetivo de este estudio es analizar y comparar los elementos valorativos asociados a la ACTITUD — categoría semántica de la teoría de la valoración (Martin, 2003; Martin \& Rose, 2003; White, 2001)dentro del discurso sobre la maternidad adolescente en noticias digitales de Venezuela y Francia. Los análisis muestran el predominio de las valoraciones negativas. En el subcorpus de noticias y comentarios en prensa venezolana ( $\mathrm{SNCV}$ ), la mayor cantidad de valoraciones tuvo lugar en la sección de comentarios, mientras que en el subcorpus de noticias y comentarios en prensa francesa (SNCF) fue en la noticia. En AFECTO, prevalece DESCONTENTO e INFELICIDAD en el SNCV, mientras que en el SNCF, DESCONTENTO e INSEGURIDAD. En JUICIO, TENACIDAD (-) e INTEGRIDAD (-) tienen mayor número de ocurrencias en el SNCV y en el SNCF son TENACIDAD (-) y NORMALIDAD (-). Por su parte, la subcategoría de APRECIACión más relevante resultó BALANCE (-) en ambos subcorpus.

Palabras clave: teoría de la valoración; categoría semántica; noticias y comentarios digitales; análisis del discurso digital 


\section{Abstract}

This investigation analyzes and compares appraisal-related elements that express ATTITUDE - Appraisal Theory's semantic category (Martin, 2003; Martin \& Rose, 2003; White, 2001) - in the discourse of Venezuelan and French digital news about teenage pregnancy. Our analysis show the prevalence of negative values. In the subcorpus of the Venezuelan digital news and comments on teenage pregnancy (SNCV), most valuations occurred in the comment section, while in the subcorpus of French digital news and comments on teenage pregnancy (SNCF), valuations were contained within the news text itself. Under the AFFECT category, DISCONTENT and UNHAPPINESS are more common in the SNCV, while DISCONTENT and INSECURITY are found in the SNCF. Under JUDGEMENT, the subcategories TENACITY (-) and ADEQUACY (-) were more frequent in the SNCV, while TENACITY (-) and NORMALITY (-) were more frequent in the SNCF. Finally, BALANCE (-), which belongs to the APPRECIATION category, was the most frequent subcategory in both subcorpus.

Keywords: Appraisal Theory; semantic category; digital news and comments; digital discourse analysis 


\section{Introducción}

En la prensa digital, las noticias y las opiniones de los lectores han sido en los últimos años objeto de análisis de diferentes disciplinas, entre ellas la lingüística. Uno de los temas tratados por la prensa que ha generado amplia polémica, debido a posturas ideológicas irreconciliables y juicios de valor, es el de la maternidad adolescente.

En Venezuela, el embarazo adolescente representa un asunto prioritario de salud pública. Según el Fondo de Población de las Naciones Unidas (UNFPA, por sus siglas en inglés), con base en el informe sobre el Estado de la población mundial de 2011, "Venezuela encabeza la tasa de embarazos precoces (sic) de Suramérica con una tasa de 101 nacimientos por cada 1.000 mujeres de 15 a 19 años [...] solo superada en Centroamérica por Honduras (108) y Nicaragua (109)" (UNFPA/EFE, 2011: para. 2). Sin duda, esta situación repercute en el habla cotidiana. Basta encender la televisión o la radio, leer el periódico o escuchar las conversaciones familiares para encontrarse con apelativos como niña madre, carajita preñada, chamita con barriga, niña preñadota, entre otros. Estos juicios pueden asociarse con la pobreza en el imaginario colectivo, y posicionar a la madre adolescente, como indica García, "en una triple condición de desigualdad: por ser mujeres, ser jóvenes y ser pobres" (1999: 244).

La abundancia de juicios de valor en torno a la maternidad adolescente nos lleva a preguntarnos, ¿la maternidad adolescente es evaluada de forma negativa en Venezuela? ¿Cómo se evalúa en otras culturas como, por ejemplo, la francesa? ¿En qué se asemejan y distinguen estas evaluaciones? Este estudio, tomado de una investigación más amplia, ${ }^{1}$ tiene como objetivo analizar y comparar los elementos valorativos asociados al dominio semántico de la ACTITUD (White, 2001; Martin \& White, 2005) dentro del discurso sobre la maternidad adolescente en noticias digitales de Venezuela y Francia. Con este fin se constituyó un corpus dividido en dos partes: un subcorpus de noticias y comentarios en pren-

1 Este estudio forma parte de la investigación realizada como trabajo de grado intitulado "Actitudes en el discurso en torno a la maternidad adolescente en prensa digital venezolana y francesa", que se presentó públicamente en 2014 dentro del programa de Maestría en Lingüística de la Universidad de Los Andes, Venezuela. 
sa venezolana (SNCV) y un subcorpus de noticias y comentarios en prensa francesa (SNCF).

Consideramos que comparar noticias digitales venezolanas y francesas puede contribuir al desarrollo de la competencia intercultural del especialista en lenguas extranjeras, en este caso específico, del aprendiz venezolano de la lengua y culturas francesas. Este tipo de estudio contrastivo invita a los aprendices a traspasar las fronteras gramaticales y a adentrarse en el ámbito social, despegándose de la visión cliché o idealizada que se ofrece comúnmente en los cursos de lenguas extranjeras. Asimismo, la construcción de concepciones culturales debe proyectarse al ámbito de la enseñanza de la traducción. No cabe duda de la necesidad de abordar la lengua como vehículo cultural, pero también como moldeadora de identidades y subjetividades.

El estudio se realizó con textos tomados de la prensa digital, lo que permitió observar el discurso desde dos perspectivas: la de la prensa misma y la del lector-usuario, quien reacciona ante la información publicada y tiene la libertad de expresar dicha reacción en forma de comentario.

En los siguientes apartados se presenta en $\$ 2$ el enfoque teórico que nos permitió abordar el estudio, esto es, la teoría de la valoración (TDV) y el dominio semántico de ACTITUD junto con sus tres categorías: AFECTO, JUICIO y APRECIACIón. En §3 se describe la metodología empleada, así como la composición del corpus recogido y las fases de análisis. Posteriormente, en $\S 4$ se muestran algunos de los resultados más significativos que nos permiten llegar a las conclusiones de nuestro estudio.

\section{Marco teórico}

\subsection{Teoría de la valoración}

Los fundamentos de la teoría de la valoración (en adelante, TDV) fueron desarrollados en la Escuela de Sídney a partir de los trabajos de Martin (2003), Martin y Rose (2003, 2008), White (2001, 2003, 2004), Martin y White (2005) y Hood y Martin (2005). Estos autores redimensionan las nociones de función interpersonal y de tenor de la lingüística sistémico funcional (LSF; Halliday, 2004) al profundizar el estudio de variantes contextuales y de negociación en 
las relaciones sociales. De acuerdo con esta teoría, el lenguaje ofrece recursos de valoración que permiten negociar y mantener las relaciones con los otros y, al mismo tiempo, expresar juicios y emociones, adoptar posiciones, hacer evaluaciones y, en general, manifestar discursivamente la actitud lingüística (White, 2004: para. 1).

La TDV, entonces, opera en el plano discursivo-semántico del lenguaje, por lo que cada una de sus categorías puede manifestarse de manera explícita - a través de elementos léxicos y gramaticales - o implícita —a través de metáforas, ironías o juegos de palabras.

\subsection{Sistemas de valoración de la TDV}

Martin y White indican que "el uso evaluativo del lenguaje tiene como función establecer (a) un posicionamiento actitudinal, (b) un posicionamiento dialógico y (c) un posicionamiento intertextual" (citados en Kaplan, 2004: 59), estos posicionamientos corresponden a tres dominios semánticos: COMPROMISO, ACTITUD y GRADACIÓN. La categoría COMPROMISO (o FUENTE) se refiere a la relación entre el enunciado y su emisor o emisores, de tipo monoglosia o heteroglosia. Por su parte, la ACTITUD se refiere a la expresión de afectos, juicios y apreciaciones. Por último, la GRADACión se refiere al grado de intensidad de los enunciados, a la fuerza misma que tiene la valoración y que determina su alcance, por lo que se toma en cuenta la FUERZA y el FOco.

Si bien la TDV fue propuesta por Martin, White y sus colaboradores de la Escuela de Sídney a través de investigaciones orientadas hacia el inglés, se han realizado estudios en diferentes ámbitos y en distintas lenguas. En América Latina destacan estudios de Argentina (Gallardo \& Ferrari, 2009), Chile (Oteiza y Pinuer, 2012), entre otros. En Venezuela se subrayan los aportes en los campos de evaluación del diálogo y la confrontación en medios de comunicación (Kaplan, 2004, 2007; Londoño, 2007; Bolívar, 2009; Olivieri, 2009) y de la identidad (López \& Álvarez, 2008). Sin embargo, la maternidad adolescente no ha sido examinada desde la teoría de la valoración. 


\subsubsection{El sistema de valoración de la ACTITUD}

El sistema evaluativo de la ACTITUD se refiere "a los valores que los hablantes comunican en sus juicios y las respuestas emocionales o afectivas que asocian con los participantes y los procesos" (White, 2001: 2). Además, se tratan las emociones, los juicios y apreciaciones positivas o negativas que puedan estar relacionados con patrones culturalmente aprendidos. La ACTITUD se manifiesta a través de tres categorías relativas al sentimiento: el AFECTO, el JUICIO y la APRECIACIÓN (White, 2004), las cuales pueden ocurrir de manera explícita o implícita. Con respecto a la valoración explícita, Kaplan establece que esta puede presentarse a través de una cualidad, un proceso o un comentario, lo que equivale al uso de adjetivos calificativos, construcciones verbales y algunas expresiones lingüísticas fijas, respectivamente (2007: 62). En la Figura 1 se muestra un resumen de las categorías pertenecientes al eje de la ACTITUD de la TDV.

\subsubsection{La categoría de AFECTO}

La primera categoría del componente de la ACTITUD es el AFECTO, el cual consiste en el conjunto de sentimientos o emociones, positivos o negativos, que los hablantes manifiestan a través de su discurso, con referencia a sí mismos, a otras personas, a objetos o circunstancias. Tal como expresan Gallardo y Ferrari, la inclusión de expresiones de afecto permite que el emisor se posicione de forma explícita o implícita con respecto a su enunciado: "cuando un autor atribuye emociones a un actor social (los obreros, los políticos, los inmigrantes) puede provocar en el lector [o receptor] una respuesta de empatía o de antipatía hacia ese actor social" (2009: 3). Martin (2003) distingue tres grandes variables de respuesta emocional, a saber: SEGURIDAD/INSEGURIDAD, SATISFACCIÓN/DESCONTENTO y FELICIDAD/INFELICIDAD. Asimismo, el AFECTO puede ser de carácter autoral o no autoral, es decir, expresar emociones propias o ajenas.

\subsubsection{La categoría de JUICI0}

El JUICIO comprende significados que sirven para evaluar positiva o negativamente el comportamiento humano en relación con un conjunto de reglas y 


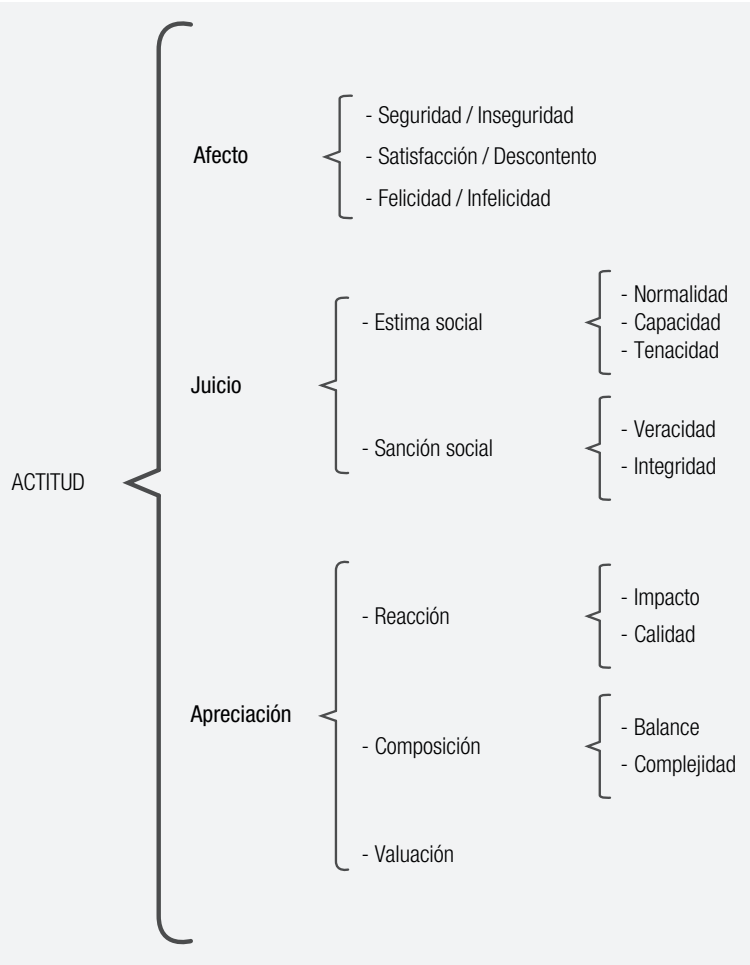

FIgURA 1. Adaptación de esquema de categorías y subcategorías de ACTITUD (Martin \& White, 2005)

también de expectativas sociales. En esta categoría de la ACTITUD se observan creencias, ideologías, representaciones sociales y culturales que cada quien tiene de su realidad (Martin \& White, en López \& Álvarez, 2008: 69). Por ello, un mismo hecho es susceptible de recibir diferentes juicios de acuerdo con la posición ideológica de la persona o el grupo social que lo juzga. White (2003) propone dos subcategorías de JUICIO: 1) JUICIO DE ESTIMA SOCIAL y 2) JUICIO DE SANCIÓN SOCIAL. Estos se subdividen, a su vez, en dimensiones positivas y negativas.

El JUICIO DE ESTIMA SOCiAL determina el grado en el que una persona se encuentra adaptada a su medio social: los aspectos positivos se admiran y los as- 
pectos negativos se critican en forma de faltas leves. Estas faltas se asocian a los ejes de la NORMALIDAD, la CAPACIDAD y la TENACIDAD.

El JUICIO DE SANCIÓN SOCIAL se relaciona con lo que es considerado correcto e incorrecto según las reglas de la sociedad a la cual pertenece un individuo, por lo que se asocia con faltas graves de tipo moral e, incluso, de tipo legal. Estas faltas se asocian con los ejes de la VERACIDAD y la INTEGRIDAD.

\subsubsection{La categoría de APRECIACIÓN}

La APRECIACIÓN comprende las valoraciones sobre objetos y constructos. En esta categoría, los sujetos solo pueden ser evaluados "cuando son considerados más como entidades que como participantes" (White, 2001: 16). La valoración de este tipo puede realizarse a partir de parámetros estéticos o no estéticos y se organiza en torno a tres variables: 1) REACCIÓN (IMPACTO y CALIDAD), 2) COMPOSICIÓN (BALANCE Y COMPLEJIDAD) y 3) VALUACIÓN.

\section{Metodología}

\subsection{Corpus del estudio}

Este estudio se basa en un corpus creado con fines específicos, que se caracterizó por ser textual, periódico, bilingüe y comparable (McEnery \& Wilson, 2001). Su representatividad no se fundamenta en el número de palabras, sino en el periodo de recolección de los datos del año 2011. Por esta razón, el tamaño de cada subcorpus (noticias de Venezuela, SNCV, y Francia, SNCF) depende del índice de aparición de noticias relacionadas en cada país y no busca una equivalencia numérica entre estos. Para la construcción del corpus, se emplearon buscadores internos de prensa digital de Venezuela y Francia. Se usaron los siguientes descriptores: embarazo precoz, embarazo adolescente, embarazo no deseado, maternidad precoz, maternidad adolescente, madre joven, madre adolescente, adolescente madre, joven madre, niña madre (Venezuela) y grossesse précoce, grossesse non désirée, maternité précoce, mère adolescente, mère jeune, jeune mère (Francia). Las fuentes del corpus son los siguientes periódicos digitales: El Universal (U) y Últimas Noticias (L) para Venezuela; L'Humanité (H), Le 
Télégramme (T), La Croix (C), L'Express (E), Le Parisien (P), Nice-Matin (N) y L'Union-Presse (I), ${ }^{2}$ para Francia. La diferencia entre la cantidad de fuentes usadas en un país y otro se debió a que, en el momento del estudio, en Francia se presentaba una mayor oferta digital de periódicos de alcance nacional y regional, mientras que en Venezuela se ofrecían menos periódicos digitales con características semejantes. Posterior al acopio de información, se hizo una depuración de la muestra a partir de la revisión de contenidos. En total, se constituyó un corpus de siete (7) noticias venezolanas y diez (10) francesas, incluyendo la sección de comentarios realizados por los usuarios que reaccionaron a las noticias presentadas. Estas noticias son textos auténticos que se muestran tal y como fueron publicados, por lo que no se aplicaron enmiendas ortotipográficas a fin de no influir sobre el corpus y su posterior análisis.

De las siete noticias digitales venezolanas, cinco presentaron reacciones de sus usuarios, con un total de 104 comentarios. De las diez noticias digitales francesas, cuatro mostraron comentarios de lectores, con un total de 26. El Cuadro 1 presenta detalladamente el conjunto de noticias que componen ambos subcorpus: el de noticias y comentarios de la prensa venezolana (SNCV) y francesa (SNCF).

CuAdro 1. Noticias componentes del sncv y del SNCF

\begin{tabular}{|c|c|c|c|}
\hline Código en CORPus & TITULO DE LA NOTICIA & $\begin{array}{l}\text { FECHA } \\
2011\end{array}$ & $\begin{array}{c}\text { NúmERO } \\
\text { DE COMENTARIOS }\end{array}$ \\
\hline E01-US & $30 \%$ son madre solteras & $07 / 05$ & 0 \\
\hline E02-US & Embarazo adolescente en Venezuela & 09/09 & 0 \\
\hline E03-UC & Misión hijos de mi pueblo Venezuela protegerá a madres adolescentes & $25 / 11$ & 29 \\
\hline E04-UC & Gobierno anuncia la Misión hijos de mi pueblo Venezuela & $25 / 11$ & 53 \\
\hline E05-US & Los embarazos adolescentes frenan el desarrollo social & $15 / 12$ & 0 \\
\hline E06-LC & Venezuela: el país con más embarazos adolescentes & $23 / 12$ & 18 \\
\hline E07-LC & Inició formación para campaña contra el embarazo precoz & $28 / 12$ & 4 \\
\hline F01-IC & Manque d'information chez les ados et conséquences désastreuses & $14 / 02$ & 5 \\
\hline F02-EC & Les mineures avortent de plus en plus & 07/03 & 5 \\
\hline F03-HS & Contraception gratuite pour les lycéens franciliens & $27 / 04$ & 0 \\
\hline
\end{tabular}

2 Las siglas que acompañan a los periódicos son las utilizadas para su identificación en la codificación del corpus. 
CuAdro 1. Noticias componentes del sncv y del sncF (continuación)

\begin{tabular}{clcc}
\hline \multirow{2}{*}{ Código EN CORPUS } & \multicolumn{1}{c}{ Título DE LA NOTICIA } & FECHA & NúmeRo \\
& & 2011 & DE COMENTARIOS \\
\hline F04-PS & Nos écrans font la part belle aux ados enceintes & $18 / 09$ & 0 \\
F05-PS & A la télé, les mamans précoces sont partout & $18 / 09$ & 0 \\
F06-NC & Pourquoi autant de grossesses précoces & $14 / 10$ & 12 \\
F07-TS & Grossesse chez l'adolescente. Progression des IVG & $19 / 11$ & 0 \\
F08-CS & A l'âge du lycée, elles sont méres & $13 / 12$ & 0 \\
F09-CS & Quelques chiffres sur les grossesses précoces & $13 / 12$ & 0 \\
F10-UC & Vers un meilleur accès à la contraception et à l'IVG pour les mineures & $14 / 12$ & 4 \\
\hline
\end{tabular}

A cada noticia del corpus le fue asignado un código diseñado para facilitar su identificación y análisis (véase Figura 2).
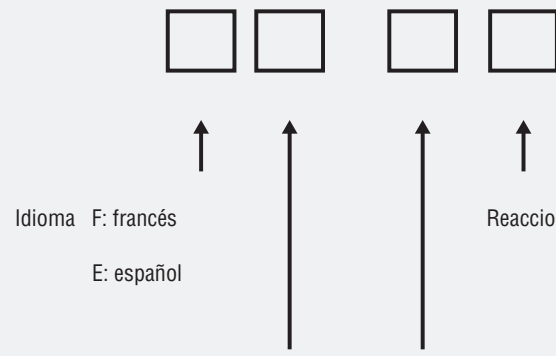

Número asignado en el corpus

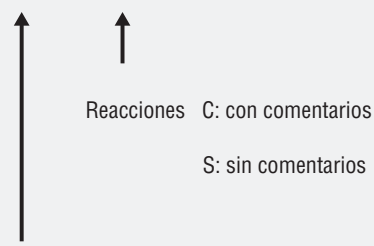

Código del periódico

Figura 2. Codificación de las noticias que componen el corpus

\subsection{Instrumentos de análisis}

Se elaboraron fichas y matrices como instrumentos para el análisis. Se diseñaron dos tipos de fichas: las primeras, para el vaciado de valoraciones explícitas e implícitas en noticias y comentarios en su contexto de aparición $(n=26)$, y las segundas, para organizar estas valoraciones por subcategorías $(n=26)$. El Cuadro 2 muestra el vaciado de elementos valorativos de NORMALIDAD hallados en el SNCV (extracto) y organizados en el segundo tipo de ficha mencionada. 
Actitud sobre la maternidad adolescente a partir de artículos de prensa digital 115

CUADRo 2. Ejemplar de ficha vaciada con valoraciones pertenecientes a la subcategoría de NoRMALDAD en el SNCv (extracto)

\begin{tabular}{|c|c|}
\hline & POSITIVO \\
\hline \multirow[t]{2}{*}{ EXPLÍCITO } & $\begin{array}{l}\text { Es normaly deseado en este pais que una niña a los } 15 \text { años tenga un bebé como signo de feminidad. } \\
\text { Juicio: estima social: Normalidad (+) [C-E02-UC] }\end{array}$ \\
\hline & $\begin{array}{l}\text { la población adolescente (la gran mayoria) de la que estamos hablando crece en casas donde eso es lo } \\
\text { común y lo natural. } \\
\text { Juicio: estima social: Normalidad (+) [C-E02-UC] }\end{array}$ \\
\hline IMPLÍ́CTOO & Sin ocurrencias \\
\hline \multicolumn{2}{|r|}{ NEGATIVO } \\
\hline EXPLÍCITO & Sin ocurrencias \\
\hline \multirow[t]{2}{*}{ IMPLÍCITO } & $\begin{array}{l}\text { Niñas que hace poquito jugaban a las muñecas, llevan ahora sobre sus brazos estos hermosos, reales y } \\
\text { pesados juguetes. } \\
\text { Juicio implícito: Normalidad (-) [C-E03-UC] }\end{array}$ \\
\hline & $\begin{array}{l}\text { así se evitará el nacimiento de bebés que llevarían una infancia no siempre afortunada que haya mujeres } \\
\text { menores de edad que pierdan su juventud por un embarazo no deseado. } \\
\text { Juicio implícito: Normalidad (-) [C-E07-LC] }\end{array}$ \\
\hline
\end{tabular}

Las matrices se emplearon para vaciar las valoraciones explícitas y luego acometer los análisis en función de las categorías gramaticales más representativas: sustantivos, adjetivos, verbos, adverbios y frases hechas. Se elaboró una matriz por cada noticia y por cada sección de comentarios. En total se obtuvieron veintiséis $(=26)$ matrices de análisis: siete de noticias y cinco de comentarios para el SNCV, por un lado; diez de noticias y cuatro de comentarios para el SNCF, por el otro. El Cuadro 3 exhibe una muestra de las valoraciones explícitas halladas en noticias y vaciadas en una matriz.

CuAdRo 3. Ejemplar de matriz vaciada con elementos valorativos explícitos de ACTitud [N-E01-US]

Título de la noticia: $30 \%$ son madres adolescentes

Código: E01-US

Fecha: 07/05/2011

Tipo de texto: Noticia

Actitud positiva: 0

Actitud negativa: 2
Actitud explícita: 1

Actitud implícita: 1

\begin{tabular}{|c|c|c|c|}
\hline Afecto & No: $x$ & $\begin{array}{l}\text { Si: } \\
\text { Cantidad: }\end{array}$ & $\begin{array}{l}\text { Afecto explícito } 0 \\
\text { Afecto implícito: } 0\end{array}$ \\
\hline
\end{tabular}

$\begin{array}{lllll}\text { Subcategoría Sustantivo Adjetivo Verbo Adverbio Frase hecha } & \text { S }\end{array}$

Seguridad $(+)$

Inseguridad (-) 
CuAdro 3. Ejemplar de matriz vaciada con elementos valorativos explícitos de Actitud [N-E01-US] (continuación)

Título de la noticia: $30 \%$ son madres adolescentes

Código: E01-US

Fecha: 07/05/2011

Tipo de texto: Noticia

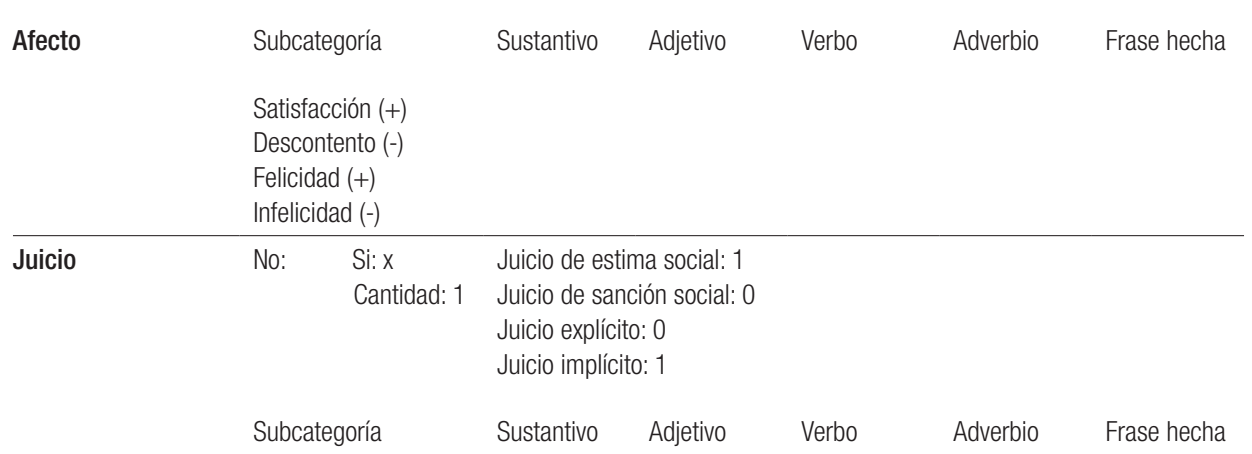

\begin{tabular}{|c|c|c|c|c|c|c|c|}
\hline Estima social & $\begin{array}{l}\text { Norm } \\
\text { Norm } \\
\text { Capa } \\
\text { Capa } \\
\text { Tenac } \\
\text { Tenac }\end{array}$ & $\begin{array}{l}\operatorname{lad}(+) \\
\operatorname{lad}(-) \\
\operatorname{ad}(+) \\
\operatorname{ad}(-) \\
\text { d (+) } \\
\text { ad (-) }\end{array}$ & & & & & \\
\hline Sanción social & $\begin{array}{l}\text { Verac } \\
\text { Verac } \\
\text { IntegI } \\
\text { IntegI }\end{array}$ & $\begin{array}{l}d(+) \\
d(-) \\
d(+) \\
d(-)\end{array}$ & & & & & \\
\hline Apreciación & No: & $\begin{array}{l}\text { Si: } x \\
\text { Cantidad: } 1\end{array}$ & $\begin{array}{l}\text { Apreciación } \\
\text { Apreciación }\end{array}$ & $\begin{array}{l}\text { kplícita: } 1 \\
\text { plícita: } 0\end{array}$ & & & \\
\hline & Subc & goría & Sustantivo & Adjetivo & Verbo & Adverbio & Frase hecha \\
\hline Reacción & $\begin{array}{l}\text { Impa } \\
\text { Impa } \\
\text { Calid } \\
\text { Calid }\end{array}$ & & & & & & \\
\hline Composición & $\begin{array}{l}\text { Balan } \\
\text { Balan } \\
\text { Comp } \\
\text { Comp }\end{array}$ & $\begin{array}{l}(+) \\
(-) \\
\text { dad (+) } \\
\text { dad (-) }\end{array}$ & & precoz & & & \\
\hline Valuación & $\begin{array}{l}\text { Valua } \\
\text { Valua }\end{array}$ & $\begin{array}{l}n(+) \\
\text { n (-) }\end{array}$ & & & & & \\
\hline
\end{tabular}




\subsection{Procedimiento}

El tratamiento de los datos incluye procedimientos de recolección y análisis de tipo cualitativo y cuantitativo; por tanto, este estudio es de tipo mixto. Los pasos que se siguieron fueron de depuración, identificación, clasificación, análisis cualitativo, análisis cuantitativo y comparación.

\section{Resultados}

Este apartado comienza con un resumen de las distinciones halladas en cuanto a la valoración positiva y negativa, a su manifestación explícita o implícita y a su aparición en noticias o comentarios. Luego se exponen los resultados de la comparación en las subcategorías de AFECTO, JUICIO y APRECIACIÓN. De las subcategorías de ACTITUD estudiadas, se exponen solo aquellas que por cantidad de ocurrencias se destacaron como las más representativas. Se analizó un total de 392 valoraciones de ACTITUD, repartidas en 243 halladas en el SNCV y 149 en el SNCF. La Figura 3 muestra el número total de valoraciones positivas y negativas observadas por categorías en ambos subcorpus.

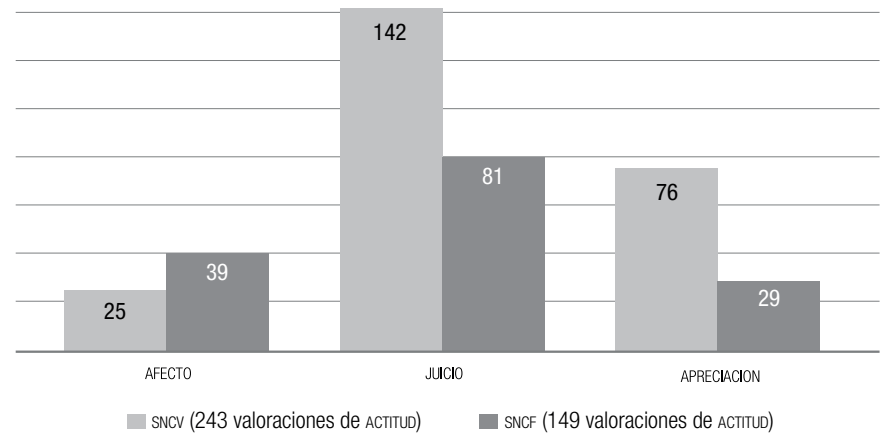

FIgURA 3. Total de valoraciones por categoría de ACTITUD en ambos corpus

Se observa en el corpus ( $\mathrm{SNCV}+\mathrm{SNCF})$ una tendencia hacia la valoración negativa en las tres categorías de ACTITUD. Con respecto a su manifestación, se ob- 
servó que las categorías AFECTO y APRECIACIÓN presentan una tendencia a ser explícitas, mientras que la de JUICIO tiende a manifestarse de forma implícita en ambos subcorpus. Con referencia a las marcas explícitas, observamos la elevada ocurrencia del adjetivo calificativo — categoría gramatical más frecuente en las tres categorías de ACTITUD - en la expresión de la valoración. Así se puede observar en (1) y (2). ${ }^{3}$

(1) Es verdaderamente dolorosa y asombrosa la cantidad de casos de embarazo precoz (sobretodo, en menores de quince años) que vemos en las calles de Venezuela. [C-E03-UC]

(2) La grossesse des jeunes filles est lourde de conséquence.

'El embarazo en las jovencitas tiene graves consecuencias.' [C-F06-NC]

En el SNCV, el adjetivo encabezó la expresión de AFECTO, mientras que para el JUICIO y la APRECIACIÓN se observó una mayor frecuencia de sustantivos valorativos. Un ejemplo de ello se muestra en (3), clasificado como un juicio negativo de INTEGRIDAD (-).

(3) que pena que en nuestra bella y rica patria tengamos esos males. [C-E03-UC]

Se destaca, además, que la mayor cantidad de ocurrencias de ACTITUD tuvo lugar en la sección de comentarios, mientras que en el SNCF, la mayor ocurrencia se observó en el texto de la noticia. Esta diferencia podría explicarse debido al bajo número de comentarios en las noticias francesas, aspecto que, en un sentido más amplio, evidenciaría una diferencia cultural con respecto a Venezuela, bien por la relevancia del tema en ambos países, bien por distinciones en el rol del periódico digital como medio de comunicación y expresión.

3 Para los fines de los ejemplos presentados, se marcaron en cursivas los términos valorativos explícitos hallados en el corpus. En el caso de las valoraciones implícitas, se marcó en cursivas todo el enunciado. 


\subsection{Comparación de AFECTO}

El AFECTo es la categoría con el índice más bajo de frecuencia en el corpus: 64/392 ocurrencias, repartidas en 25/243 para el SNCV y 39/149 para el SNCF. Las expresiones de AFECTO en el SNCF se manifiestan, casi de forma equivalente, en valoraciones AUTORALES (11/25) y NO AUTORALES (14/25), mientras que en el SNCF se refleja una clara preferencia para expresar valoraciones NO AUTORALES (32/39). En (4) se muestra un ejemplo de valoración de AFECTO autoral en el SNCV, mientras que en (5) se muestra un ejemplo de valoración de AFECTO no autoral hallada en el SNCF.

(4) el trauma de las niñas adolescentes embarazadas lo llevan en el corazón. [C-E02-UC]

(5) C'est en effet un phénomène qui augmente", regrette la vice-présidente de Gynécologie sans frontière, Florence Francillon.

'Es de hecho un fenómeno que aumenta, lamenta la vicepresidenta de Ginecología sin fronteras, Florence Francillon.' [N-F02-EC]

Las subcategorías INFELICIDAD $(13 / 25)$ y DESCONTENTO $(8 / 25)$ resultaron las más frecuentes en el SNCV, mientras que en el SNCF se observó con igual cantidad de ocurrencias SATISFACCIón (12/39) y DESCONTENTO (12/39). En el ejemplo (6), el adverbio lamentablemente representa en forma explícita y autoral la valoración de INFELICIDAD hallada en el SNCV.

(6) pero lamentablemente la poblacion adolescente (la gran mayoria) de la que estamos hablando crece en casas donde eso es lo comun y lo natural. [C-E02-UC]

El ejemplo (7) ilustra la valoración de DESCONTENTO en forma explícita y autoral en el SNCV.

(7) que pena que en nuestra bella y rica patria tengamos esos males y que ahora lejos de atacarlos para eliminarlos simplemente se fomentan...que indignacion, dios. [C-E03-UC] 
En el ejemplo (5), antes mostrado, se puede observar una manifestación explícita de DESCONTENTO, mientras que la subcategoría SATISFACCIón del mismo subcorpus se refleja en (8).

(8) l'enfant peut apparaître comme un sujet de réussite, un libre choix, une manière de donner un sens à leur existence', explique Fize, sociologue au CNRS.

'el niño puede aparecer como un motivo de éxito, una elección libre, una forma de darle sentido a la propia existencia, explica Michel Fize, socióloga del CNRS.' [N-F08-CS]

\subsection{Comparación de Juıcı}

En ambos subcorpus el JUICIO resultó la categoría con mayor índice de aparición respecto de las otras, con un total de 223 valoraciones repartidas en 142/243 juicios en el SNCV y 81/149 de juicios en el SNCF. Las subcategorías de JUICIO con mayor cantidad de valoraciones en el SNCV pertenecen a TENACIDAD (-) e INTEGRIDAD (-). Los ejemplos (9), (10) y (11) muestran estas subcategorías. El ejemplo (9) contiene una valoración de TENACIDAD (-) en el SNCV.

(9) No es ignorancia, ni inocencia, ni descuido, para muchas es simple SINVERGUENZURA [C-E02-UC]

Los ejemplos (10) y (11) evidencian el juicio de INTEGRIDAD negativa, expresado en ambos casos de forma implícita en comentarios.

(10) Una adolescente quema sus posibilidades de estudio y laborales si sale embarazada [C-E02-UC]

(11) a lo mejor nuestras jóvenes [venezolanas] no están dispuestas a realizarse abortos, a diferencia de otros países [N-E02-UC]

En cuanto al SNCF, resultaron relevantes las subcategorías de TENACIDAD (-) y de NORMALIDAD (-). Los ejemplos (12) y (13) dan cuenta de la TENACIDAD, mientras que el ejemplo (14) es indicativo de la NORMALIDAD. 
(12) Pour se créer un compte sur Facebook, alimenter un skyblog là pas de problème, mais la contraception est trop compliquée.

'para abrirse una cuenta en Facebook o mantener un Skyblog no tienen ningún problema, pero usar anticonceptivos, eso sí se les hace demasiado complicado.' [C-F10-PC]

(13) Beaucoup de jeunes filles démontrent une impressionnante méconnaissance de leur corps.

'Muchas jóvenes demuestran un sorprendente desconocimiento de sus propios cuerpos.' [N-F02-EC]

(14) les grossesses désirées à l'âge du lycée restent pour le moment marginale. 'los embarazos deseados en edad de liceo siguen siendo, hasta el momento, marginales.' [N-F08-CS]

\subsection{Comparación de APRECIACIÓN}

La APRECIACIÓN presentó 105 valoraciones en total, repartidas en 76/243 en el SNCV y 29/149 en el SNCF. La subcategoría de APRECIACIÓN más relevante en ambos subcorpus resultó la de BALANCE (-), como se observa en el ejemplo (15), seguida en el SNCV por la de IMPACTO (-), como en (16).

(15) Naturalmente, las adolescentes de los estratos sociales pobres están más expuestas a los embarazos precoces especialmente si existen círculos viciosos de madres adolescentes en sus familias [C-E02-UC]

(16) Es alarmante nuestro nivel de embarazo en adolescentes entre 15 y 19 años [N-E02-UC]

En el SNCF, la segunda subcategoría relevante resultó ser la de COMPLEJIDAD (-), seguida de IMPACTO (+). Los ejemplos (17) y (18) muestran BALANCE (-) у COMPLEJIDAD (-) en el SNCF:

(17) même si l'éducation sexuelle à l'école est loin d'être suffisante, ce n'est qu'une partie du problème. 
'aunque la educación sexual en la escuela está lejos de ser suficiente, esto no es más que una parte del problema.' [N-F02-EC]

(18) Pourtant, elle représente un enjeu sociétal majeur et peut, si la situation n'est pas abordée, aboutir à des situations très complexes de grossesses précoces.

'Sin embargo, [la relación entre las jóvenes y los anticonceptivos] representa un asunto social importante y puede, si no se enfrenta, dar lugar a situaciones muy complejas de embarazos adolescentes.' [N-F01-IC]

El ejemplo (19) permite apreciar una valoración de IMPACTO (+), subcategoría también relevante en el SNCF.

(19) ces maternités à l'âge du lycée exercent une certaine fascination.

'estas maternidades en edad de liceo ejercen una especie de fascinación.' [N-F08-CS]

\section{Discusión y conclusiones}

La maternidad adolescente es un tema vigente que da cuenta de un problema multifactorial enfrentado por la población, principalmente, en países latinoamericanos. Este problema ha sido abordado y estudiado por especialistas de diferentes ámbitos; sin embargo, parece que desde la linguiística han sido muy pocos los aportes. Este estudio tuvo como objetivo describir y analizar el discurso sobre la maternidad adolescente que hacen la prensa digital y los usuarios-lectores de noticias en dos países culturalmente disímiles: Venezuela y Francia. El corpus del estudio se limitó a textos del año 2011. En consecuencia, para establecer tendencias generales se tendría que ampliar a otros corpus de estudio y a otros tipos de texto que faciliten el análisis del discurso en torno a este tema.

Dentro de la variedad de voces del corpus, que se expresan tanto en los textos de las noticias como en los comentarios, observamos que prevalece un discurso hegemónico valorizante que dicta pautas y se ciñe a ciertas creencias sobre el deber ser de las adolescentes. Esta es una tendencia que se refleja desde los mismos apelativos utilizados para referirse al fenómeno en cuestión. Aunque se usen términos no valorativos en ambos subcorpus, como embarazo adolescente/maternité précoce, prevalece el uso de términos que encierran una carga 
valorativa negativa, tales como embarazo precoz (SNCV), grossesse précoce y grossesse non désirée ( $\mathrm{SNCF}$ ).

Al analizar la ACTITUD presente en el discurso sobre maternidad adolescente en noticias digitales de Venezuela y Francia, saltan a la vista semejanzas y diferencias halladas sobre este aspecto en ambas culturas. Si nos referimos a las semejanzas, notamos que en ambos subcorpus prevalece el discurso negativo donde el mayor peso de valoración recae sobre la adolescente, a quien se le insta de manera constante a seguir un comportamiento específico en cuanto al deber ser en su paso de la infancia a la juventud, determinado casi totalmente por su postura ante las relaciones sexuales y la concepción. Organizaciones internacionales como la Organización Mundial de la Salud (oms) o el Fondo de las Naciones Unidas para la Infancia (UNICEF) se muestran como portavoces de un discurso que, difundido a través de los medios de comunicación y entes estatales de ambos países, presenta aseveraciones que trazan el moldeamiento de la concepción de la joven mujer en ambas culturas.

La adolescente francesa/la madre adolescente francesa. La joven adolescente francesa es dibujada en el corpus de estudio como una joven irresponsable en la mayoría de los casos, cuya actitud varía entre la ingenuidad de la niña que no conoce el funcionamiento de su cuerpo y la inconsciencia de la adolescente que actúa sin pensar en las consecuencias de sus actos. Aunque el discurso refleja una cultura que toma en cuenta la capacidad de elección de la joven sobre su vida sexual y reproductiva, no deja de mostrar una tendencia claramente enfatizada hacia el rol y la responsabilidad de la mujer, a través de críticas y demarcaciones sobre su deber ser. Así, el actor social abordado en los textos es criticado por su bajo rendimiento escolar, por tener relaciones conflictivas con sus padres y por no presentar perspectivas claras de futuro. También se nos muestra a la joven que esconde su embarazo para poder preservarlo, con el fin de llamar la atención de sus padres, o como medio para escapar de un hogar conflictivo o para intentar ganar el respeto social que, considera, de otra manera no conseguiría. Respecto a la madre adolescente, observamos que este actor social escapa del centro del debate en el SNCF. Pareciera que en dichos textos el tema se aborda únicamente desde la perspectiva de la adolescente, quien decide ser madre a pesar de su edad. Las valoraciones se concentran en el conjunto de razones que la llevan a tomar la decisión de no practicarse una interrupción voluntaria del embarazo (IVG, en 
francés). Luego de tomada la decisión de continuar el embarazo, el tema sale de las discusiones porque, de hecho, no se observaron rasgos que construyeran la identidad de la madre adolescente francesa.

La adolescente venezolana/la madre adolescente venezolana. La joven adolescente venezolana es dibujada en los textos que componen el SNCV con una fuerte carga de críticas y condenas que la responsabilizan de arruinar su propia infancia y juventud, pero también de provocar y acentuar problemas sociales en Venezuela. En el SNCV no se hallaron enunciados sobre la prefiguración de la adolescente como sujeto que puede asumir la sexualidad de manera responsable. Para la adolescente venezolana, la lista del deber ser es larga; la norma social le dicta esperar antes de iniciarse en la vida sexual activa, emplear métodos de protección, saber escoger sus parejas y tener relaciones estables, formarse en el estudio y tener proyectos de vida.

En el SNCV se trata constantemente la importancia de la prevención; sin embargo, una vez que se ha dado la concepción, se espera que esa joven irresponsable se convierta en madre responsable. Así, se convierte en sujeto de otras valoraciones que ponen en duda su capacidad para ejercer adecuadamente el rol materno. Entonces, se insta a la joven a hacerse responsable de su hijo, a continuar con sus estudios, a no representar un gasto económico para los demás (padres, gobierno), es decir, a cumplir con expectativas casi imposibles de alcanzar de forma paralela y a tan corta edad. Además, se le responsabiliza de graves problemas sociales como el aumento de la pobreza y la delincuencia. En definitiva, sus posibilidades de ser actor social no criticado son prácticamente nulas.

Como puede observarse a través de los resultados, la TDV proporciona instrumentos para la observación y análisis de un conjunto amplio de elementos valorativos que forman parte de cualquier discurso. De la misma manera, es innegable su utilidad para identificar elementos discursivos que construyen identidades sociales, así como para deshilvanar el tejido de subjetividades halladas en un tema y luego dejar ver la influencia de la cultura en estos mismos elementos. Su aplicación es útil para el lingüista, ya que permite organizar los elementos de lenguaje evaluativo que, de otra manera, podrían quedar solapados si se utilizaran otros métodos de análisis lingüístico.

No obstante, es importante señalar algunas limitaciones halladas al aplicar dicho modelo en este estudio. En primer lugar, resultó complicado analizar de- 
terminados enunciados valorativos según una u otra categoría, debido a la dificultad para clasificar las emociones, los sentimientos y, en general, las posturas. Este problema se presentó debido a la búsqueda de la objetividad en los análisis dentro de un marco donde resulta prácticamente imposible excluir el juicio del investigador, elemento que traspasa la identificación y categorización de los enunciados durante los análisis. Para ilustrar esta dificultad, retomamos el ejemplo (10): "Una adolescente quema sus posibilidades de estudio y laborales si sale embarazada" [C-E02-UC]. Este enunciado fue clasificado durante los análisis como una INTEGRIDAD negativa; es decir, un asunto que representa la gravedad en su nivel más alto dentro de la escala de JUICIO negativo. El hecho de que una adolescente pueda perder la oportunidad de estudiar y trabajar al quedar embarazada podría considerarse un asunto importante o trivial, y esta categorización dependerá de que el investigador lo considere una falta grave o leve, si este hecho va en contra de lo correcto o lo esperado y si concibe este comportamiento como criticable o condenable. Aquí se demuestra que es imposible llegar a una conclusión única sin que esta decisión sea traspasada por nuestro propio juicio y postura ética y moral.

Así pues, resulta complejo determinar si un comportamiento es loable o criticable sin pasar por los filtros social y personal, tal como puede observarse en el ejemplo (11): "a lo mejor nuestras jóvenes [venezolanas] no están dispuestas a realizarse abortos, a diferencia de otros países" [N-E02-UC]. El Cuadro 4 presenta algunas de las posibilidades de clasificación que podrían aplicarse a (11), acompañadas de un conjunto de posibles argumentos que se enmarcan en la cultura venezolana tal y como la muestran las noticias y los comentarios incluidos en el SNCV.

Cuadro 4. Posibilidades de clasificación de la valoración en el ejemplo (11)

\begin{tabular}{ll}
\hline INTEGRIDAD (+) & Loable. La vida del nonato es su derecho inalienable. No realizarse un aborto es virtuoso y además, se \\
enmarca en la legalidad, ya que no está permitido abortar (en Venezuela). La maternidad es sagrada.
\end{tabular}

TENACIDAD (+) Admirable. Asumir la maternidad en la adolescencia es símbolo de responsabilidad y valentía. 
Cuadro 4. Posibilidades de clasificación de la valoración en el ejemplo (11) (continuación)

\begin{tabular}{ll}
\hline TENACIDAD (-) & $\begin{array}{l}\text { Criticable. Asumir la maternidad en la adolescencia es un acto irresponsable, ya que se carece de capa- } \\
\text { cidades psicológicas y económicas para el desempeño de la misma y es incluso un riesgo para la vida } \\
\text { de la joven y del bebé. }\end{array}$ \\
NORMALIDAD (+) & $\begin{array}{l}\text { No llama la atención. En Venezuela, lo común es que las venezolanas no estén dispuestas a abortar. } \\
\text { NORMALIDAD (-) }\end{array} \quad \begin{array}{l}\text { Llama la atención. La actitud de las jóvenes venezolanas no es normal con respecto la situación "en otros } \\
\text { países" donde abortar es un derecho de la mujer. }\end{array}$ \\
\hline
\end{tabular}

Si bien no resulta contradictorio que una valoración pueda clasificarse en distintas categorías a la vez, se demuestra la dificultad para llegar a la exhaustividad y a una clasificación que se aleje de los filtros personales del analista.

Otra limitación observada fue el hallazgo de algunos vacíos en la clasificación de valoraciones de ACTITUD propuesta por la TDV. Debido a la gran cantidad de emociones, sentimientos y posturas intersubjetivas, se hallaron enunciados cuyo análisis no se reflejaba completamente en ninguna de las subcategorías propuestas, por lo que fue necesario proponer su clasificación dentro de los parámetros establecidos. Si bien puede darse a lo largo de la clasificación, en el presente estudio se evidenció en la subcategoría IMPACTO. Kaplan dice que "la reacción [categoría que encierra IмРАсто] describe cuánto atrajo nuestra atención el proceso, objeto o texto evaluado y de qué manera nos impactó" (2004: 66). La respuesta positiva supone algo cautivador, llamativo o atractivo, mientras que la negativa se asocia con algo aburrido, tedioso o predecible. Surge entonces la pregunta: ¿cómo clasificar algo que llame la atención si es negativo, por ejemplo, un escándalo? En este contexto, se pueden presentar enunciados como (16): "Es alarmante nuestro nivel de embarazo en adolescentes entre 15 y 19 años" [N-E02-UC].

En (16) se observa la evaluación de un constructo, esto es, del número de embarazos de adolescentes en Venezuela, lo que ubica a la oración en la categoría de APRECIACIÓN. El adjetivo alarmante nos lleva a asociarlo directamente con IMPACTO, pero allí mismo se tiene que estas cifras no son ni 'cautivadoras' (IMPACto positivo) ni ‘aburridas' (IмPACTo negativo). Se clasificó al adjetivo como negativo, puesto que prevalece el sentido negativo acerca del embarazo adolescente. Las diez valoraciones negativas clasificadas en esta subcategoría del corpus SNCV presentaron la misma situación, lo que muestra una tenden- 
cia en el corpus y la consecuente necesidad de crear una subcategoría adecuada para clasificarlo.

En este sentido, la TDV y su sistema de valoración de ACTITUD obliga a plantearse hasta qué punto o bajo qué justificaciones es válido establecer nuevas subcategorías, y si ello no haría de la propuesta de análisis un conjunto enorme de subcategorías inmanejables para los fines prácticos de una investigación. Ante estos contratiempos, surgen algunos elementos que se pueden considerar claves para el uso apropiado de la TDV. El primero es la importancia del contexto, no solo de los elementos valorativos explícitos, sino también de los enunciados valorativos. El estudio del texto permite obtener una visión de la tendencia general de su autor y del engranaje de las valoraciones presentes, ya que las mismas no suelen presentarse de forma aislada, sino que se encuentran en consonancia con un discurso, ayudando a construir un punto de vista y, claro está, una intencionalidad. De allí la importancia vital de que el investigador tenga conocimiento del contexto sociocultural en el que están inmersos los textos estudiados. En segundo lugar, resulta primordial una revisión exhaustiva del estado del arte, ya que permite ver la clasificación de elementos hechos por otros autores, determinar las tendencias y observar nuevas corrientes en torno a la TDV en contextos discursivos específicos.

La riqueza de los datos aportados en este estudio puede generar futuras investigaciones. El formato del texto virtual constituye un terreno fértil para diversas aproximaciones. Una de ellas podría orientarse a la valoración expresada a través de caracteres tipográficos, tales como el uso de las comillas y las mayúsculas. El caso del uso de las mayúsculas valorativas puede observarse en el ejemplo (9), donde juega un rol de intensificador del discursivo valorativo. Elementos de este tipo permiten al lingüista estudiar nuevos rasgos acerca del lenguaje y su uso en los canales actuales de comunicación.

Todos estos aspectos pudieran sumarse a la construcción de una posible gramática evaluativa, tal y como sus máximos exponentes lo han manifestado. Sin embargo, su elaboración supondría grandes esfuerzos, dado que los elementos explícitos forman grupos que, por su naturaleza, solo pueden ser abiertos, al igual que las valoraciones implícitas contenidas en enunciados, las cuales resultan aún más difíciles de catalogar. En virtud de ello, se considera que, en lugar de una meta tan ambiciosa, pudiera ser de mayor provecho y utilidad la cons- 
trucción de gramáticas específicas a un campo preciso de evaluación, en un tipo de discurso determinado y con una variedad lingüística particular.

\section{Referencias}

Bolívar, Adriana (2009). "Democracia” y "revolución” en Venezuela: un análisis crítico del discurso político desde la lingüística de corpus. Oralia: Análisis del Discurso Oral, 12, 27-54.

Gallardo, Susana, \& Ferrari, Laura (2009). Análisis del discurso de los médicos: cómo valoran su salud y su profesión. IntraMed. Recuperado de http://www.intramed.net/UserFiles/archivos/Analisis\%20de1\%20discurso $\% 20$ de $\% 201$ os\%20médicos_\%20como\%20valoran\%20 su\%20salud\%20y\%20su\%20profesión.pdf

García Hernández, Gloria Elizabeth (1999). Un enfoque social sobre el embarazo en la adolescencia. Iztapalapa. Revista de Ciencias Sociales y Humanidades, (47), 235-248. Recuperado de http://148.206.53.234/revistasuam/iztapalapa/include/getdoc.php?id=633\& article $=645 \&$ mode $=$ pdf

Halliday, Michael (2004). An introduction to functional grammar. Londres: Arnold. Recuperado de http://www.uel.br/projetos/ppcat/pages/arquivos/RESOURCES/2004_HALLIDAY_ MATTHIESSEN_An_Introduction_to_Functional_Grammar.pdf

Hood, Susan, \& MARTin, James R. (2005). Invocación de actitudes: el juego de la gradación de la valoración en el discurso. Revista Signos, 38(58), 195-220.

KaPlan, Nora (2004). Nuevos desarrollos en el estudio de la evaluación en el lenguaje: la teoría de la valoración. Boletín de Lingüística, 22, 52-78.

KAPLAN, Nora (2007). La teoría de la valoración: un desarrollo de los estudios sobre la evaluación en el lenguaje. En Adriana Bolívar (Comp.), Análisis del discurso: ¿por qué y para qué? (pp. 63-86). Venezuela: Los libros de El Nacional. Recuperado de https://books.google.com.mx/ books?id=V6Y3Fppu5WEC\&printsec=frontcover\&hl=es\&source=gbs_ge_summary_r\& $\mathrm{cad}=0 \# \mathrm{v}=$ onepage $\& \mathrm{q}=$ caraca $\& \mathrm{f}=$ false

Londoño Zapata, Óscar IvÁn (2007). Los estudios del discurso en Venezuela y América Latina. Un compromiso social, político y académico. Entrevista a la Dra. Adriana Bolívar. Discurso \& Sociedad, 1(4), 68-700. Recuperado de http://www.dissoc.org/ediciones/v01n04/ DS1\%284\%29Londono.pdf

López, Carmen Aracelys, \& Álvarez, Alexandra (2008). Valoración e identidad en el discurso de sujetos con síndrome de Down. Lengua y Habla, 12, 64-78. 
Martin, JAmEs (2003). Introduction [Special Issue: Negotiating heteroglossia: Social perspectives on evaluation]. [Versión electrónica]. Text, 23(2), 171-181. Recuperado de http://www. grammatics.com/appraisal/textspecial/martin-intro.pdf

Martin, JAmes, \& Rose, DAVID (2003). Working with discourse: Meaning beyond the clause. Londres: Continuum.

Martin, James, \& Rose, David (2008). Genre relations: Mapping culture. Londres: Equinox.

Martin, James, \& White, Peter (2005). The language of evaluation: Appraisal in English. Hampshire: Palgrave Macmillan.

McEnery, Tony, \& Wilson, Andrew (2001). Corpus linguistics: An introduction. Edimburgo: Edinburgh University Press.

Olivieri Pacheco, Giannina (2009). Análisis de la construcción discursiva presente en los artículos de opinión de la prensa venezolana tras las elecciones presidenciales de 2006. Anuario Ininco, 21(1), 193-216.

Oteiza, Teresa, \& Pinuer, Claudio (2012). Prosodia valorativa: construcción de eventos y procesos en el discurso de la historia. Discurso \& Sociedad, 6(2), 418-446.

UNFPA/EFE. (2011, 23 de diciembre). Venezuela: el país con más embarazos adolescentes. Últimas noticias, sección Salud.

White, Peter R. R. (2001). Un recorrido por la teoría de la valoración (teoría de la valoración) (Trad. por Elsa Ghio). Birmingham: Universidad de Birmingham.

White, Peter R. R. (2003). Beyond modality and hedging: A dialogic view of the language of intersubjective stance. Text, 23(2), 259-284. Recuperado de http://www.prrwhite. info/prrwhite, $\% 202003, \% 20$ Beyond $\% 20$ modality $\% 20$ and $\% 20$ hedging $\% 20-\% 20$ A $\% 20$ dialogic\%20view\%20of\%20the\%20language\%20of\%20intersubjective\%20stance.pdf

White, Peter R. R. (2004). An introductory tour through appraisal theory. [Versión electrónica]. Recuperado de http://www.grammatics.com/appraisal/appraisaloutline/framed/ appraisaloutline.htm 